\title{
Evolution of Optimal 3D placement of UAV with Minimum Transmit Power
}

\author{
Asad Mahmood ${ }^{1, ~ *, ~ M u h a m m a d ~ Q a m a r ~ U s m a n ', ~ K h u r r a m ~ S h a h z a d ~}{ }^{1}$, Nayab Saddique ${ }^{2}$ \\ ${ }^{1}$ Department of Electrical and Computer Engineering, Comsat University Islamabad, Wah Cantt, Pakistan \\ ${ }^{2}$ Department of Electrical Engineering, Capital University of Science and Technology, Islamabad, Pakistan \\ Email address: \\ Asad10125@hotmail.com (A. Mahmood), engrqamarusman@gmail.com (M. Q. Usman), khurram.sanwal@gmail.com (K. Shahzad), \\ nayyabmughal883@gmail.com (N. Saddique) \\ ${ }^{*}$ Corresponding author
}

\section{To cite this article:}

Asad Mahmood, Muhammad Qamar Usman, Khurram Shahzad, Nayab Saddique. Evolution of Optimal 3D placement of UAV with Minimum Transmit Power. International Journal of Wireless Communications and Mobile Computing. Vol. 7, No. 1, 2019, pp. 13-18. doi: $10.11648 /$ j.wcmc.20190701.12

Received: February 3, 2019; Accepted: March 8, 2019; Published: March 29, 2019

\begin{abstract}
Due to vast technology advancement in communication System, it has been possible to use unmanned aerial vehicle (UAV) that can fly independently and worked remotely without carrying any human personnel. UAV provides new methods for communication in military and civilian application. They have small operating and installation cost. They provide flexible way for communication. Unmanned aerial vehicle can be used as Wireless base station in cellular system providing an alternative communication mechanism for cellular communication in any disaster or emergency situation when existing terrestrial network goes down. However besides these several advantages UAVs has many Unique design challenges for both indoor and outdoor users. Energy limitation is one of the major challenges as each UAV has limited battery source so due to limited battery time completing the task in minimum Hover time is another challenge. Another major challenge is Optimal UAVs placement in such a way that Sum data rate of all the users is maximized. this paper investigate the problem of Optimal UAVs placement and minimum transmit power through which better signal to noise ratio is achieved for indoor users, first proved our problem is mixed integer non- linear problem and our objective function is convex and use Genetic algorithm and Nomad to solve our problem, Through Numerical result conclude that optimal placement of UAVs is achieved with minimum path loss and minimum transmit power required to cover indoor user.
\end{abstract}

Keywords: Optimal Placement, Path Loss, NOMAD, Minimum Transmitted Power

\section{Introduction}

Unmanned air vehicle is aircraft which can be operated without the interference of human it can be programmed as per our requirement. UAV's also known as Drones in modern world. Recent technologies are developing these UAV's for different purposes. These drones have their own onboard chip which can be programmed as per requirements. Onboard chips can be controlled remotely. Researchers are working with UAV's for different goals. UAV's provides wireless communications between users in different scenarios [1]. It assists the terrestrial base station BS if some disaster happens i.e. base station failure. UAV's can be deployed rapidly where the demand increase. With a lot of advantages there are also some constraints which are discuss in later section. UAV's have on board electronics components so they required some power for their operation. For meeting their power requirement use batteries [1]. As mentioned above that there are some constraints which limits over goal. Here batteries limit Hover time or flight time which is the biggest challenge in UVA's communication [1-2]. 


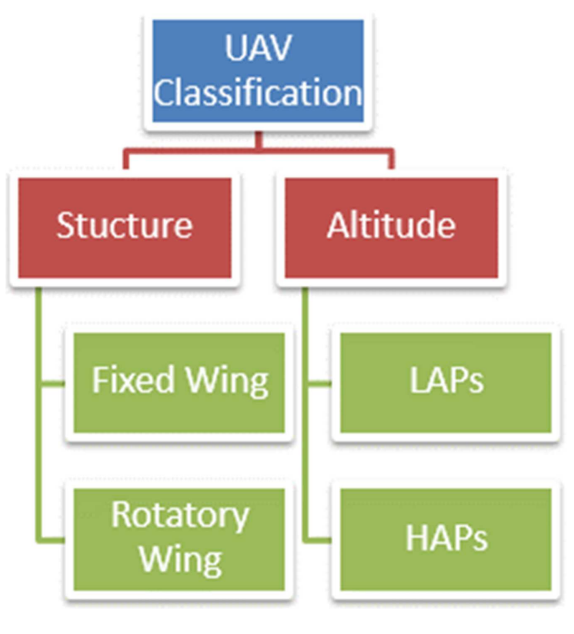

Figure 1. UAV Classification.

There are usually two types of UAV's as shown in Figure 1 w.r.t their altitudes Low Altitude Platform (LAP) and High Altitude Platform (HAP) Low altitude platform are usually some meters or few kilometer above ground and they have rotary wings. The main feature of LAP which is the most beneficial is hovering. Hovering time depend upon the batteries which is the main concern for the deployment and for the researchers to extend the flight time and use minimal power or can say energy efficient. They are cost effective UAV's [3]. High altitude platform are above $17 \mathrm{~km}$ from ground they required runways for taking of they are unusually big in size. They cannot hover which is main deal with that but their flight time is much higher than LAP's [3]. If classify UVV's w.r.t Sizes then there are some types which are given as small size UAV's Micro or Nano UAV's Small size UAV's, Medium size UAV's, Large size UAV's If talk about the applications of UAV's then find that UAV's have a lot of application in our daily life [4]. UAV's application only in telecom sector then there are some applications given as Wireless communication in disaster (flood) Used As UAV-BS and interlinks with terrestrial BS for communication, UAV's deployed where the burden on base station is more UAV's used as relay in communication, Used as BS where terrestrial BS failed and there is high demand of communication [5]. Now some other applications in our daily life then some are given below [5]. UAV's used for industrials inspection and also for maintenance as well as automatic repairing. For temperature sensing For delivery products urgently (amazon) For electricity lines sensing Used in automotive sensing Used for taking images where human cannot do photography [6]. These are few applications in our daily life. define all above application in one line then can be said that UAV's can be used in surveillance, for military use search as well as rescues and in telecommunication [4-7]. As with a lot of advantages there are some technical challenges also in UAV-BS there are some constraints which become hurdle in achieving over optimal goal [8-14]. The man challenges inn UAV's are throughput, transmission rate, optimal placement in different scenarios i.e. urban, urban dense, rural, ground some big gathering like Hajj in Makkah, interference control, maximum coverage with energy efficient, path loss, channel modeling LOS or NLOS challenges. These are few basic challenges for the latest work. Here in our paper mainly focus on the optimal placement of UAV's which will discuss in later parts of paper. UAV-helped correspondences with accentuation on the utilization cases, organize engineering, channel attributes, and UAV plan contemplations [15]. Author built up a archetypal for the likelihood of Line of sight correspondence among a UAV-BS besides a collector in addition assessed the height of the UAV-BS that augments the inclusion locale [16]. On the other hand UAVBS transmits at full power and defined the 3D UAV BS situation issue as a quadratic partner compelled blended whole number non-direct issue addressed [17]. Writers built up a heuristic calculation dependent on molecule swarm advancement. The calculation sub ideally finds the base number of UAVBSs and their areas to serve a specific district. The creators streamlined the UAV-BS height that outcomes in greatest inclusion area and least transmit control for two cases, a solitary UAV-BS and two UAV-BSs in [9]. The inclusion likelihood as a component of elevation and radio wire gain is inferred. Besides, the creators built up a strategy to send various UAV-BSs dependent on circle pressing Think about two instances of useful intrigue and give proficient answers for the defined issue under these cases [9-11]. In the primary case, find the base transmit power to such an extent that an indoor client with the greatest way misfortune can be secured. In the second case, accept that the areas of indoor clients are symmetric over the elements of each floor and propose an inclination plunge calculation (optimization toolbox) to find a proficient 3D situation of a UAV. Our principle commitment in this paper is to check the issue of effective UAV placement, where the goal is to limit the all-out transmit control required to cover the tall structure, when the areas of indoor clients are consistently conveyed in each floor, and propose a molecule swarm advancement calculation for finding a productive area of the UAV.

Rest of the paper is formatted as follow, Section 2 contain system model, Section 3 contain Optimization Tools where as section 4 contain discussion about numerical results.

\section{System Model}

Let consider the scenario of disaster or emergency occur in high raised budging where terrestrial network are not available at the present time, here users $\mathrm{i}$ are randomly distributed. Dimension of high raised building $(0, \mathrm{xmax}),(0, \mathrm{ymax})$ and $(0$. zmax) are shown in Figure 3. Where (xu, yu, zu) denote the optimal location of UAV. Where $\varnothing$ is the incident angle between UAV and position of user i. Where $d 2 d$ is the $2 D$ distance between user and UAV's \& $d 3 d$ is $3 D$ distance between user and UAV's as shown in Figure 2.

$$
\begin{gathered}
d 3 d=\sqrt{\text { height }^{2}+\mathrm{d} 2 \mathrm{~d}^{2}} \\
d 2 d=\sqrt{\left(x_{u}-x_{i}\right)^{2}+\left(y_{u}-y_{i}\right)^{2}} \\
\theta=\arctan \left(\frac{\text { height }}{d 2 d}\right)
\end{gathered}
$$


Path loss in energy density of an electromagnetic signal when transmitted through transmitter in free space.

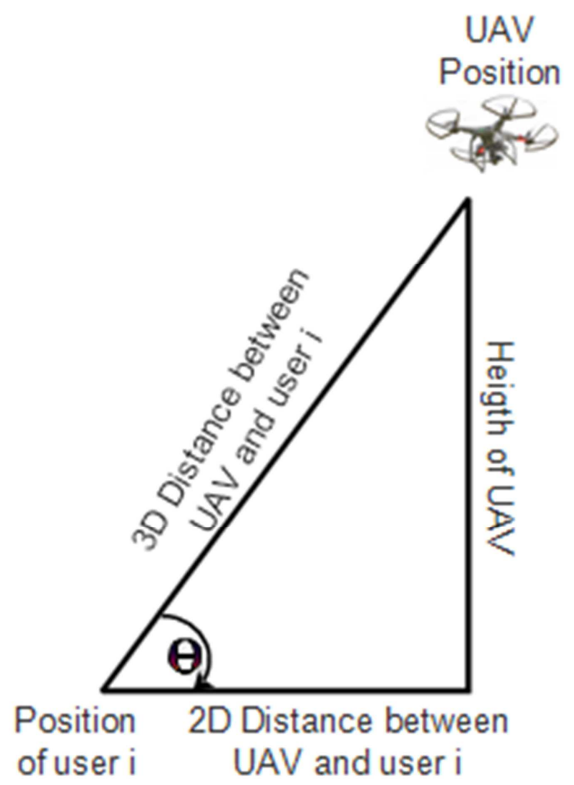

Figure 2. Parametric Triangle.

The attenuation of energy of signal when propagate through space consider as free space path loss for our above mentioned scenario path loss can be models as

$$
P L_{i}=P L_{F}+P L_{B}+P L_{I}
$$

Here $P L_{F}, P L_{B} \& P L_{I}$ are free space, buildings penetration loss and indoor losses respectively

$$
\begin{gathered}
P L_{F}=a \cdot \log _{10}(3 D d i s)_{i}+a \cdot \log _{10}\left(2 G H z+b_{1}\right) \\
P L_{B}=b_{2}+b_{3}\left(1-\cos \theta_{i}\right)^{2} \\
P L_{I}=b_{4}(2 D \text { dis })_{i}
\end{gathered}
$$

Here the above path loss formation shows that as the 2D distance increase, 3D distance also increase which lead toward decrement in the incident angle. On the other hand if 2D distance decreases 3D distance also decrease which lead toward increment in incident angle $\theta_{i}$. Where environmental coefficient are given in Table 1

Table 1. Environmental Coefficient.

\begin{tabular}{llllll}
\hline $\mathbf{a}$ & $\boldsymbol{b}_{\mathbf{1}}$ & $\boldsymbol{b}_{\mathbf{2}}$ & $\boldsymbol{b}_{\mathbf{3}}$ & $\boldsymbol{b}_{\mathbf{4}}$ & $\mathbf{f}$ \\
\hline 20 & 32.4 & 14 & 15 & 0.5 & 2 \\
\hline
\end{tabular}

In telecom sector the term Transmitted power defined as the power which is actually a transmitter giving at the output. Where the minimum power required to satisfy the rate each user or the power required to make it able for the communication during the above mentioned scenario below.

$$
P_{\min } T_{x}=\left(2^{\frac{r_{i} N}{B}}-1\right) \cdot P L_{i}
$$

$\boldsymbol{r}_{\boldsymbol{i}}=$ required rate of each user

$\mathrm{B}=$ total bandwidth available

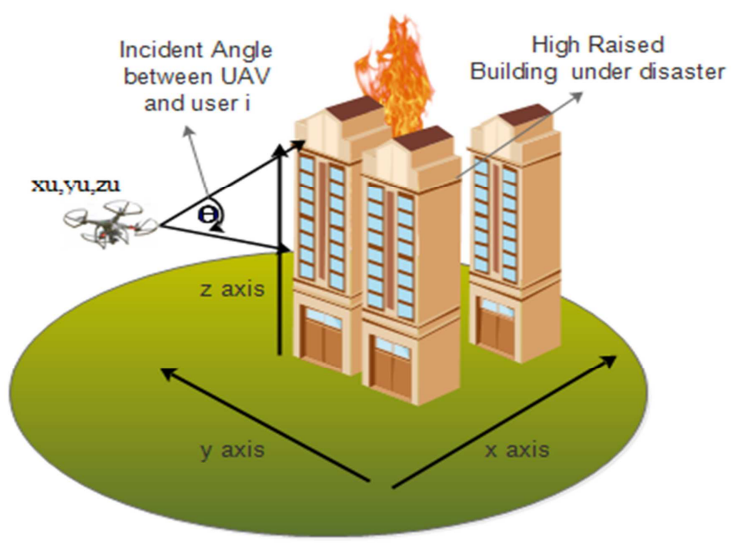

Figure 3. System Model.

Now the maximum transmitted power of UAV's is given as $P T_{\text {max }}$ and path loss represented as $P L_{\max }$

$$
P L_{\text {max }}=\frac{P T_{\text {min }}}{\left(2^{\frac{r i N}{B}}-1\right) \times \eta}
$$

Where

$\mathrm{N}=$ number of user (randomly distributed)

$\eta=$ Noise co-efficient

The problem formulated as convex nonlinear mixed integer problem. The mathematical form of above mentioned problem is given as

$$
\operatorname{Min}_{x_{u} y_{u} z_{u}} \sum_{i=1}^{N} P L_{i}
$$

Subject to

$$
\begin{gathered}
\sum_{i=1}^{N} P L_{i} \leq P L_{\max } \\
\sum_{i=1}^{N} P T_{i} \leq P T_{\max } \\
X_{\min } \leq X_{u} \leq X_{\max } \\
y_{\min } \leq y_{u} \leq y_{\max } \\
Z_{\min } \leq Z_{u} \leq Z_{\max }
\end{gathered}
$$

Here the problem in convex mix integer non liner problem. Solver to solve (MINLP) is NOMAD and compare its result with Genetic Algorithm (GA) a bench mark to solve Optimization problems.

\section{Optimization Tools}

To solve the convex mixed integer nonlinear the solver is NOMAD its result will compared with the heuristic GA (genetic algorithm) which is this case is bench mark to solve optimization problem. NOMAD is Mesh Adaptive Direct search algorithm which is implemented in object oriented language $\mathrm{C}++$. Pseudo code for NOMAD is given below in Figure 4.

The GA (genetic algorithm) is based on DARWIN's theory of natural evolution. GA algorithm inspired same heuristic search approach where the strongest mate selected for mating

For reproduction in search of offspring of next generation as shown in Figure 5. 


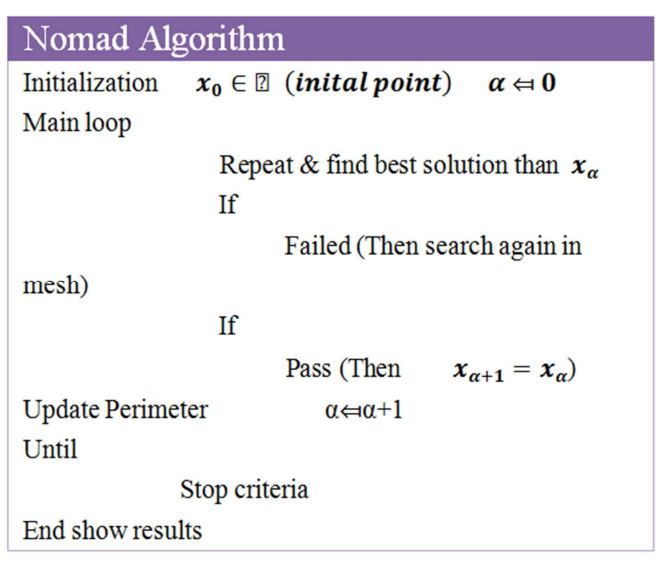

Figure 4. Nomad Phudo Code.

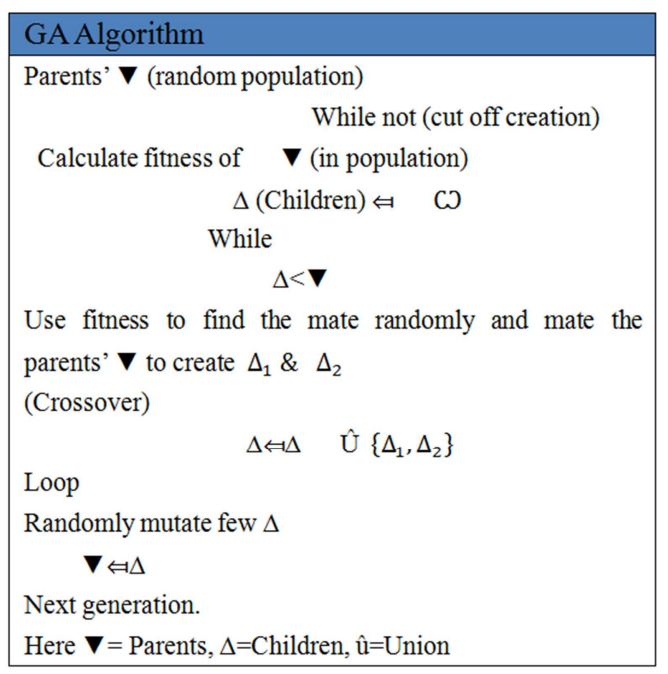

Figure 5. GA Phudo Code.

\section{Results and Discussions}

Path loss models portray the flag lessening between transmit and a get receiving wire as an element of the spread separation and different parameters. A few models incorporate numerous subtleties of the landscape profile to gauge the flag constriction, while others simply think about transporter recurrence and separation. Path Loss is the decline in encouragement that transpires as a radio wave spreads over a separation as shown in Figure 6.

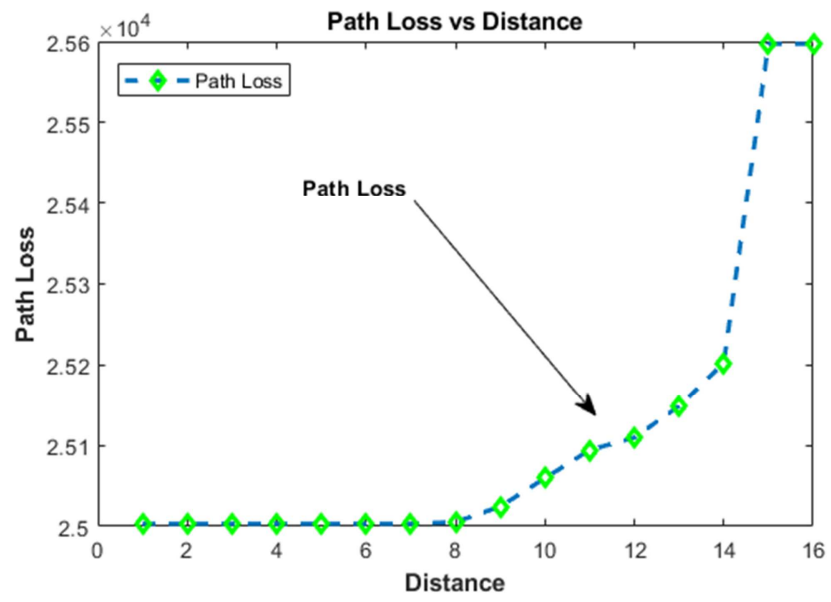

Figure 6. Power Vs Distance.

The essential factor in Path-Loss is the decline in flag quality over separation of the radio waves themselves. Radio waves pursue an inverse square law for power thickness: the power thickness is relative to the converse square of the separation. Each time you twofold the separation, you get just a single fourth the power.

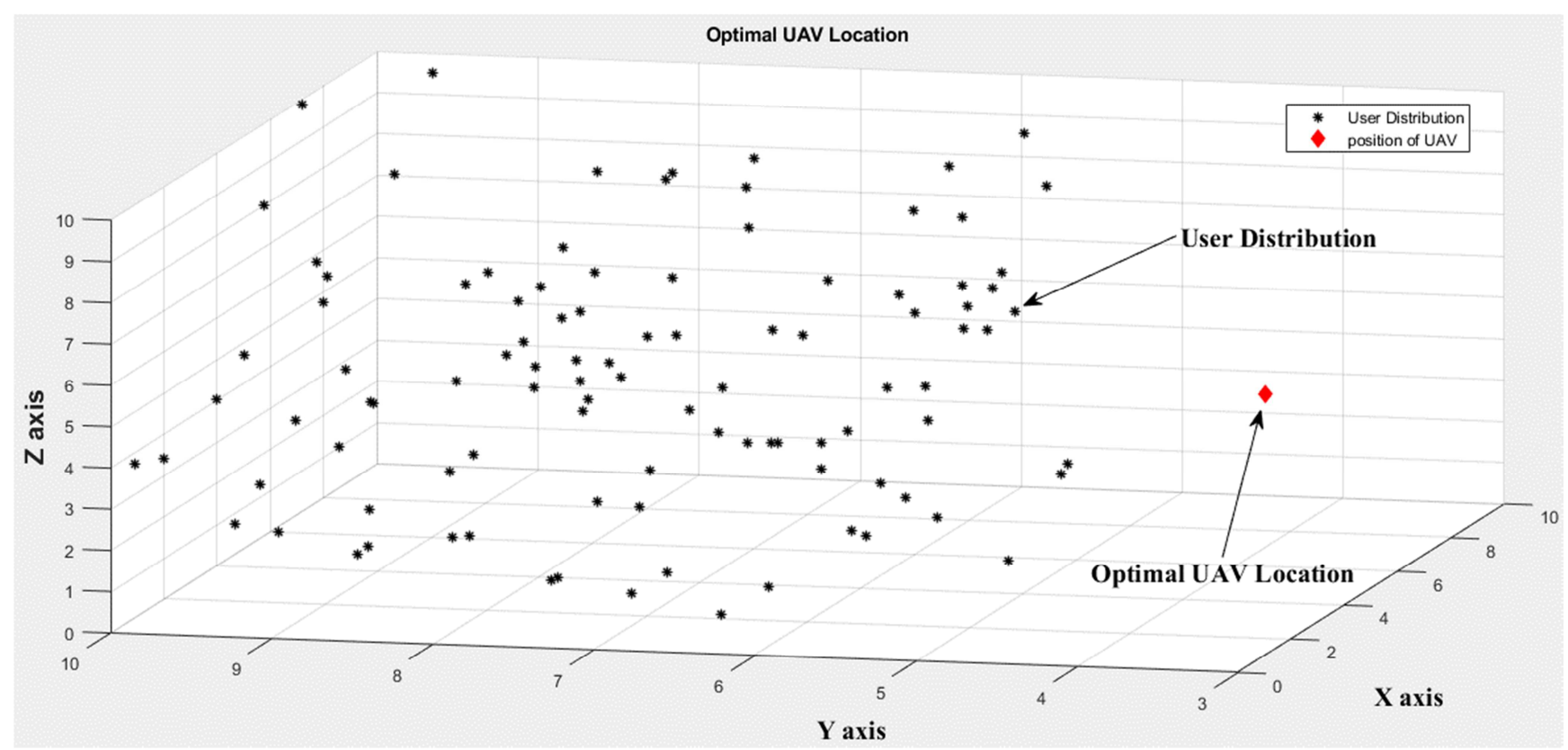

Figure 7. Optimal Location of UAV.

Convergence is an event in relation to progression. It causes growth because in reality every single person is the same. Full geographic genealogy algorithms (a variety of evolution) is simply the use of sensors (the way to combine people with a 
new generation). At the time of time the populations can be used to solve one single solution, but this solution does not have a high quality, with estimation, i.e. Population was paused. However, not necessarily centrally negative, because most of the time, after that, the stability of the program, means that the best program is all of the common ones and their behavior is similar to one another (similar to the previous generation programs). Many times the value of the vaccine is used widely. Various DNA Tactics Tenders can be avoided shown the comparative analysis between convergences of GA and build in optimizer NOMAD Figure 8 shown that NOMAD converge earlier as compared to heuristic algorithm GA Unmanned Air Vehicles (UAVs) can be used as air-conditioned base-based bets when removing the mobile network. Previous studies prior to the coverage of UAV based on UAV, especially the landowner's stationers from land users are considered to be considered by land users. This article take up the scenario of the scenario under horror cases (such as earthquakes or floods), when the cellular network is down. Problem of UAV Placement, which aims to determine the purpose of at least one call as Figure 7 shown the optimal location of UAV.

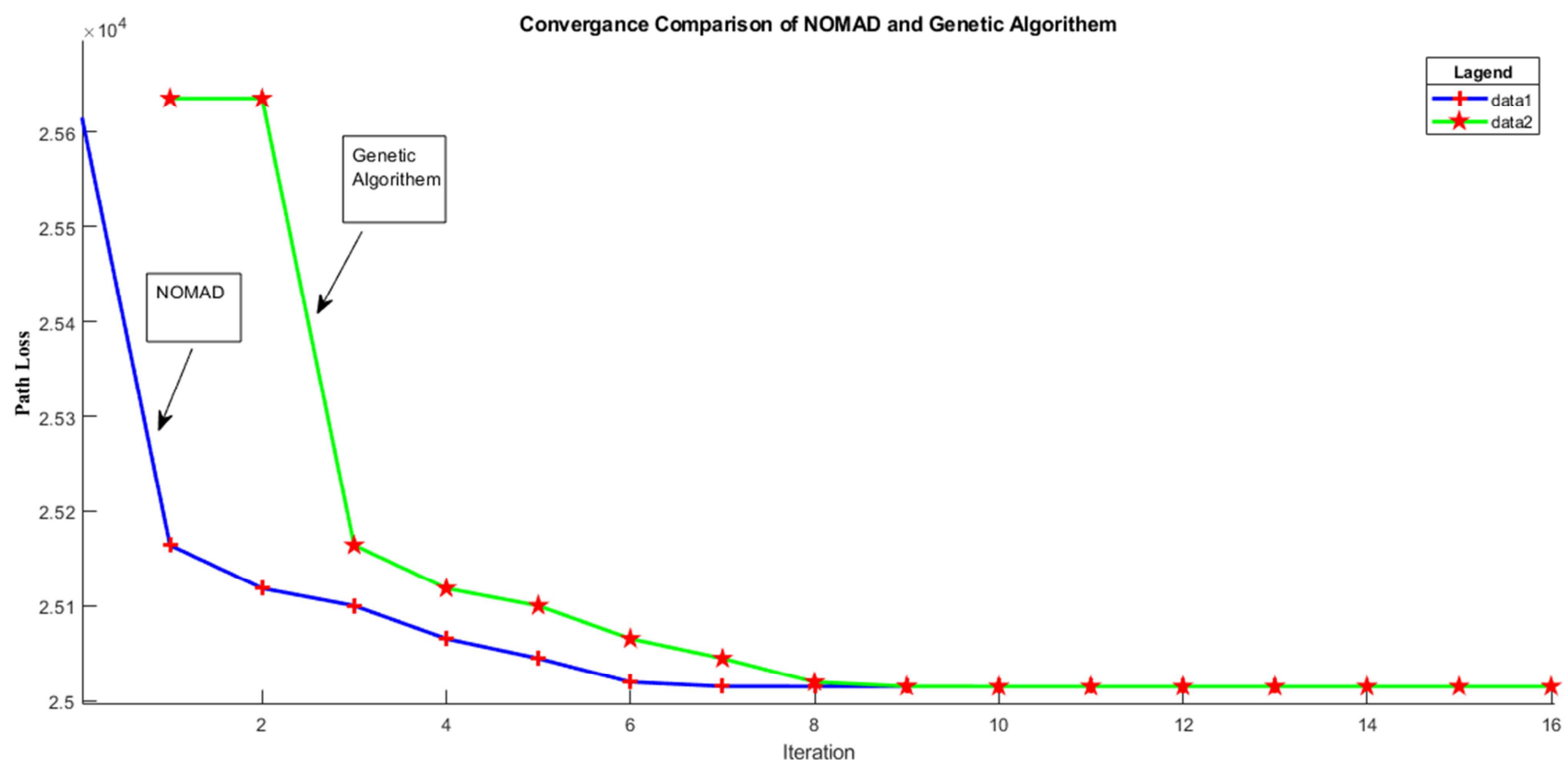

Figure 8. Comparative Analysis of Optimization Tools.

This implies each 6-dBm increment in yield control copies the conceivable separation that is attainable. Other than transmitter control, another factor influencing range is recipient affectability. It is typically communicated in $\mathrm{dBm}$. Since both yield power and collector affectability are expressed in $\mathrm{dBm}$, you can utilize straightforward expansion and subtraction to figure the most extreme Path Loss that a framework can bring about in equation (maximum path loss equation). Which mean that as the distance between transistor and receiver increase snr which result in reduction of received power as shown in Figure 9

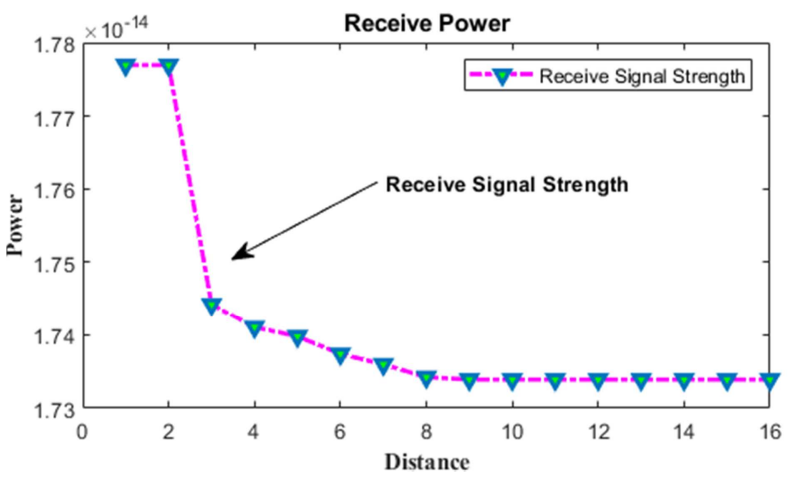

Figure 9. Power Vs Distance.

\section{Conclusion}

In any case of natural disaster, survival of victim haFve great importance, for this UAV's plays its important role enable the victim to make call to survive for this optimal placement of UAV have great importance. Optimal placement at the point that lead toward minimum path loss across all the user in a scenario. To solve this optimization problem NOMAD and GA were taken into account results shown NOMAD converge earlier as compared to $\mathrm{Ga}$ and try to find the relationship between path loss and distance and find out decrement in path loss as the distance increase, whereas transmitted power increase, if the distance between UAV and User i is optimal there will be more chance of survival of users in disaster situation.

\section{References}

[1] Y. Zeng, R. Zhang, and T. J. Lim, "Wireless Communications with Unmanned Aerial Vehicles: Opportunities and Challenges," no. May, pp. 36-42, 2016.

[2] M. Mozaffari, W. Saad, and M. Bennis, "Wireless Communication using Unmanned Aerial Vehicles ( UAVs ): Optimal Transport Theory for Hover Time Optimization," pp. 1-30. 
[3] E. Kalantari, I. Bor-yaliniz, A. Yongacoglu, and H. Yanikomeroglu, "User Association and Bandwidth Allocation for Terrestrial and Aerial Base Stations with Backhaul Considerations."

[4] M. Mozaffari, S. Member, W. Saad, and S. Member, "Unmanned Aerial Vehicle with Underlaid Device-to-Device Communications : Performance and Tradeoffs," pp. 1-32.

[5] M. Mozaffari, W. Saad, and M. Bennis, "Unmanned Aerial Vehicle with Underlaid Device-to-Device Communications: Performance and Tradeoffs," vol. 1276, no. c, pp. 1-14, 2016.

[6] J. Xu, Y. Zeng, and R. Zhang, "UAV-Enabled Wireless Power Transfer: Trajectory Design and Energy Region Characterization."

[7] M. M. Azari, F. Rosas, K. Chen, and S. Pollin, "Ultra Reliable UAV Communication Using Altitude and Cooperation Diversity."

[8] N. H. Motlagh, M. Bagaa, and T. Taleb, "UAV Selection for a UAV-based Integrative IoT Platform," 2016.

[9] A. Kumbhar, S. Singh, and M. Solutions, "UAV Assisted Public Safety Communications with LTE-Advanced HetNets and FeICIC," pp. 3-8, 2020.
[10] W. Khawaja, O. Ozdemir, and I. Guvenc, "UAV Air-to-Ground Channel Characterization for mmWave Systems," no. I.

[11] Y. Zeng, R. Zhang, and T. J. Lim, "Throughput Maximization for UAV-Enabled,” vol. 64, no. 12, pp. 4983-4996, 2016.

[12] P. Milano and D. G. Cileo, "Politecnico di Milano School of Industrial and Information Engineering," 2017.

[13] K. E. Ave, K. E. Ave, and K. E. Ave, "Theoretical Analysis of the Target Detection Rules for the UAV-based Wireless Sensor Networks," 2017.

[14] X. Lin et al., "The Sky Is Not the Limit: LTE for Unmanned Aerial Vehicles," pp. 1-7, 2017.

[15] M. Narang, S. Xiang, W. Liu, J. Gutierrez, and L. Chiaraviglio, "UAV-assisted Edge Infrastructure for Challenged Networks."

[16] V. Sharma, M. Bennis, and R. Kumar, "UAV-Assisted Heterogeneous Networks for Capacity Enhancement," pp. 1-4.

[17] R. Zhang, "Wireless Communications with Unmanned Aerial Vehicles: Opportunities and Challenges." 